\title{
Cache Aided Decode-and-Forward Relaying Networks: From the Spatial View
}

\author{
Junjuan Xia, ${ }^{1}$ Fasheng Zhou $\left(\mathbb{D},{ }^{2}\right.$ Xiazhi Lai ${ }^{10},{ }^{1}$ Haochuan Zhang, \\ Hongbin Chen $(1),{ }^{4}$ Qinghai Yang, ${ }^{5}$ Xin Liu, ${ }^{6}$ and Junhui Zhao ${ }^{7}$ \\ ${ }^{1}$ School of Computer Science and Educational Software, Guangzhou University, Guangzhou, China \\ ${ }^{2}$ School of Physics and Electronic Engineering, Guangzhou University, Guangzhou, China \\ ${ }^{3}$ Guangdong University of Technology, Guangdong, China \\ ${ }^{4}$ Key Laboratory of Cognitive Radio and Information Processing, Guilin University of Electronic Technology, Guilin, China \\ ${ }^{5}$ State Key Laboratory on ISN, School of Telecommunications Engineering, Xidian University, Xian 710071, China \\ ${ }^{6}$ School of Information and Communication Engineering, Dalian University of Technology, Dalian, China \\ ${ }^{7}$ School of Information Engineering, East China Jiaotong University, Nanchang, China \\ Correspondence should be addressed to Fasheng Zhou; zhoufs@gzhu.edu.cn
}

Received 1 November 2017; Revised 23 December 2017; Accepted 1 February 2018; Published 23 April 2018

Academic Editor: Dajana Cassioli

Copyright (C) 2018 Junjuan Xia et al. This is an open access article distributed under the Creative Commons Attribution License, which permits unrestricted use, distribution, and reproduction in any medium, provided the original work is properly cited.

We investigate cache technique from the spatial view and study its impact on the relaying networks. In particular, we consider a dualhop relaying network, where decode-and-forward (DF) relays can assist the data transmission from the source to the destination. In addition to the traditional dual-hop relaying, we also consider the cache from the spatial view, where the source can prestore the data among the memories of the nodes around the destination. For the DF relaying networks without and with cache, we study the system performance by deriving the analytical expressions of outage probability and symbol error rate (SER). We also derive the asymptotic outage probability and SER in the high regime of transmit power, from which we find the system diversity order can be rapidly increased by using cache and the system performance can be significantly improved. Simulation and numerical results are demonstrated to verify the proposed studies and find that the system power resources can be efficiently saved by using cache technique.

\section{Introduction}

With the rapid progress in wireless big data, a lot of wireless techniques have been proposed to enhance the transmission quality and reliability [1-10]. Cooperative relaying, such as amplify-and-forward (AF) and decode-and-forward (DF) relaying protocols, is an effective means to enhance network reliability as well as security $[11,12]$, extend coverage region $[13,14]$, and reduce the impact of interference [15-17]. Therefore, cooperative relaying is a significant technique in the current and future generations wireless networks, and the research on relaying networks has been active. The outage performance of multirelay networks was studied in $[18,19]$, and the exact outage probabilities were derived. In [20], the authors turned to investigate the performance of relaying networks in terms of symbol error rate (SER). In [21, 22], the performance of relaying networks in the high signal-to-noise ratio (SNR) regime was analyzed and the diversity order was revealed.

To improve the transmission performance relaying networks, cache technique has been proposed to enhance the user experience quality and reduce the latency. In particular, the authors in [23] studied the fundamental limits of caching and found an information-theoretic formulation of the caching problem by focusing on its basic structure. Then the authors in [24] applied the coding technique into cache and studied the fundamental limits by proposing an improved delivery rate-cache capacity tradeoff. Further, the authors in [25] investigated content-centric sparse multicast beamforming for cache-enabled cloud RAN and revealed 


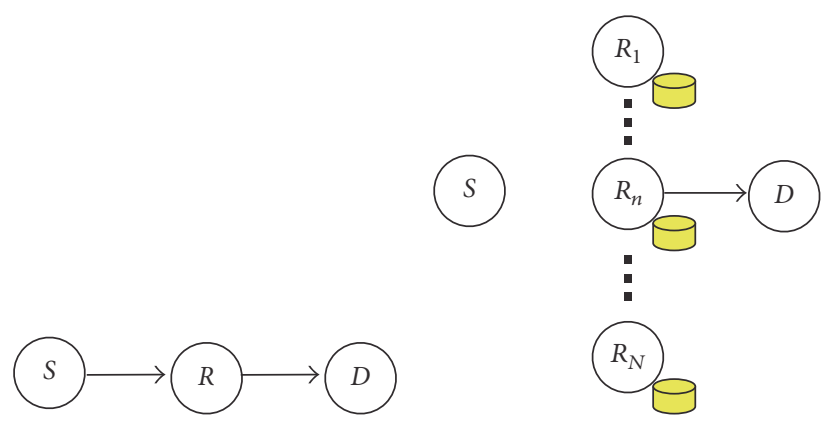

(a) Without cache

(b) With cache

FIgURE 1: Decode-and-forward relaying networks.

that caching strategy had a significant impact on the system performance. The application of cache into relaying networks has been studied in [26], where optimization of hybrid cache placement for collaborative relaying was investigated. In this paper, the signal transmission cooperation gain and content delivery diversity were achieved to enhance the system transmission performance. There are some other works on the application of cache in network security to ensure the secure information transmission [27-30]. However, to the best of the authors' knowledge, there has been little work on the spatial view of cache on performance improvement of the relaying networks.

In this work, we study the spatial view of cache on the DF relaying networks, where the source communicates with the destination with several DF relays. If the cache is used, the source can prestore the data among the nodes around the destination. In this way, the destination can directly access the data from the nearby nodes, instead of having to communicate with the source. For the traditional dualhop relaying and the DF relaying assisted by the cache, we study the system transmission performance by deriving the analytical expression of outage probability and SER. We also provide the asymptotic expressions of outage probability and SER in the high region of transmit power, from which we find that the cache can efficiently improve the system performance by increasing the transmission diversity order. Numerical and simulation results are demonstrated to verify the proposed studies in this paper.

Notations 1 . We use $\mathscr{C} \mathscr{N}\left(0, \sigma^{2}\right)$ to represent a circularly symmetric complex Gaussian random variable (RV) with zero mean and variance $\sigma^{2}$. We use $f_{X}(\cdot)$ and $F_{X}(\cdot)$ to represent the probability density function (PDF) and cumulative density function $(\mathrm{CDF})$ of the random variable (RV) $X$, respectively. In addition, the notation $X \sim \operatorname{Naka}(m, \alpha)$ represents that the random variable $X$ is subject to Nakagami- $m$ distribution, where the PDF of variable of $Y=|X|^{2}$ is $f_{Y}(y)=$ $\left(m^{m} y^{m-1} / \alpha^{m} \Gamma(m)\right) e^{-m y / \alpha}$. We use $\operatorname{Pr}[\cdot]$ to denote probability.

\section{System Model}

Figure 1(a) shows the traditional dual-hop relaying networks without cache, where a single decode-and-forward relay node
$R$ assists the two-phase data transmission from the source $S$ to the destination $D$. In contrast, Figure 1(b) demonstrates the cache aided dual-hop relaying networks, where the data can be prestored at $N$ nodes $\left\{R_{n} \mid 1 \leq n \leq N\right\}$ (the relays can obtain the data from the source during nonpeak time, and these relays can work in either the cooperative or noncooperative ways [31]) around the destination $D$. Then the data can be directly transmitted from the relays to the destination, instead of through the traditional dual-hop relaying links. Due to the size limitation, each node has a single antenna only [32-35], and we assume that each node operates in a time-division half-duplex mode [36-39]. In the following, we describe the data transmission for the relaying networks without and with cache, respectively.

For the traditional data transmission without cache as shown in Figure 1(a), let $h_{S R} \sim \operatorname{Naka}\left(m_{1}, \alpha\right)$ and $h_{R D} \sim$ $\operatorname{Naka}\left(m_{2}, \beta\right)$ denote the channel parameter of the $S-R$ and $R$ $D$ links, respectively. In the first phase, the source $S$ sends the normalized signal $s$ to the relay, and then the relay receives

$$
y_{R}=\sqrt{P} h_{S R} s+n_{R}
$$

where $P$ is the transmit power of the source and $n_{R} \sim$ $\mathscr{C} \mathcal{N}\left(0, \sigma^{2}\right)$ is the additive white noise at the relay. If the relay $R$ can correctly decode the signal from the source, it will forward the signal to the destination in the second phase, and accordingly the destination $D$ receives

$$
y_{D}=\sqrt{P} h_{R D} s+n_{D}
$$

where $n_{D} \sim \mathscr{C} \mathscr{N}\left(0, \sigma^{2}\right)$ is the additive white noise at the destination. From (1)-(2), we can obtain the received end-toend SNR at the destination $D$ as

$$
\mathrm{SNR}_{D}=\frac{P}{\sigma^{2}} \min (u, v),
$$

where $u=\left|h_{S R}\right|^{2}$ and $v=\left|h_{R D}\right|^{2}$ denote the channel gains of the first and second hops, respectively.

For the cache aided DF relaying networks demonstrated in Figure 1(b), the source data can be firstly prestored at the $N$ relay nodes around the destination, and then the data transmission can be directly performed from the relays to the destination, instead of through the traditional two-hop 
relaying links. Specifically, when the $n$th relay $R_{n}$ is used for data transmission, the received signal at the destination $D$ is given by

$$
y_{D}=\sqrt{P} h_{R_{n}, D} s+n_{D}
$$

where $h_{R_{n}, D} \sim \operatorname{Naka}\left(m_{2}, \beta\right)$ is the channel parameter of the second-hop relaying links associated with the $n$th relay. Accordingly, the received end-to-end SNR at the destination $D$ is given by

$$
\mathrm{SNR}_{D}=\frac{P}{\sigma^{2}} v_{n}
$$

where $v_{n}=\left|h_{R_{n}, D}\right|^{2}$ is the instantaneous channel gain of the $R_{n}-D$ link. Among these $N$ relays, one best relay $R_{n^{*}}$ can be selected to assist the data transmission, and the selection criterion is given by

$$
n^{*}=\arg \max _{1 \leq n \leq N} v_{n}
$$

\section{Performance Evaluation}

3.1. Outage Probability. In this part, we investigate the data transmission performance by deriving the analytical expression of outage probability as well as the asymptotic expression with high transmit power. Let $P_{\text {out }}^{\mathrm{wo}}$ and $P_{\text {out }}^{\mathrm{w}}$ denote the outage probability of the DF relaying networks without and with cache assistant, respectively.

3.1.1. Without Cache. For the traditional dual-hop relaying networks without cache, the outage event occurs when the transmission data rate is below a target data rate $R_{t}[40,41]$,

$$
\frac{1}{2} \log _{2}\left(1+\mathrm{SNR}_{D}\right)<R_{t}
$$

where the term $1 / 2$ comes from the two-phase data transmission. This equation is equivalent to

$$
\mathrm{SNR}_{D}<\gamma_{1 t}
$$

where $\gamma_{1 t}=2^{2 R_{t}}-1$ is the SNR threshold without cache. Accordingly, we can write the system outage probability $P_{\text {out }}^{\text {wo }}$ as

$$
\begin{aligned}
P_{\mathrm{out}}^{\mathrm{wo}} & =\operatorname{Pr}\left(\mathrm{SNR}_{D}<\gamma_{1 t}\right) \\
& =\operatorname{Pr}\left(\min (u, v)<\frac{\gamma_{1 t} \sigma^{2}}{P}\right) \\
& =1-\operatorname{Pr}\left(\min (u, v) \geq \frac{\gamma_{1 t} \sigma^{2}}{P}\right) .
\end{aligned}
$$

By considering that the variables of $u$ and $v$ are independent of each other, we can rewrite $P_{\text {out }}^{\text {wo }}$ as

$$
P_{\text {out }}^{\text {wo }}=1-\operatorname{Pr}\left(u \geq \frac{\gamma_{1 t} \sigma^{2}}{P}\right) \operatorname{Pr}\left(v \geq \frac{\gamma_{1 t} \sigma^{2}}{P}\right) .
$$

By applying the PDFs of $f_{u}(u)=\left(m_{1}^{m_{1}} u^{m_{1}-1} /\right.$ $\left.\alpha^{m_{1}} \Gamma\left(m_{1}\right)\right) e^{-m_{1} u / \alpha}$ and $f_{v}(v)=\left(m_{2}^{m_{2}} v^{m_{2}-1} / \beta^{m_{2}} \Gamma\left(m_{2}\right)\right) e^{-m_{2} v / \beta}$, we can compute the analytical outage probability of the DF relaying networks without cache as

$$
\begin{aligned}
P_{\text {out }}^{\mathrm{wo}} & =1-\int_{\gamma_{1 t} \sigma^{2} / P}^{\infty} \frac{m_{1}^{m_{1}} u^{m_{1}-1}}{\alpha^{m_{1}} \Gamma\left(m_{1}\right)} e^{-m_{1} u / \alpha} d u \\
& \times \int_{\gamma_{1 t} \sigma^{2} / P}^{\infty} \frac{m_{2}^{m_{2}} v^{m_{2}-1}}{\beta^{m_{2}} \Gamma\left(m_{2}\right)} e^{-m_{2} v / \beta} d v \\
= & 1-e^{-\left(\gamma_{1 t} \sigma^{2} / P\right)\left(m_{1} / \alpha+m_{2} / \beta\right)} \sum_{k_{1}=0}^{m_{1}-1} \sum_{k_{2}=0}^{m_{2}-1} \frac{1}{k_{1} ! k_{2} !} \\
& \times\left(\frac{m_{1} \gamma_{1 t} \sigma^{2}}{P \alpha}\right)^{k_{1}}\left(\frac{m_{2} \gamma_{1 t} \sigma^{2}}{P \beta}\right)^{k_{2}},
\end{aligned}
$$

where [42, eq. (3.351.2)] is applied in the last equality. The analytical outage probability in the above equation contains elementary functions only, and hence it is easy to be evaluated.

To obtain more insights on the system, we propose to derive the asymptotic expression of outage probability for the DF relaying networks without cache, in the high transmit power regime. Since the approximation of $e^{x} \simeq \sum_{n=0}^{N}\left(x^{n} / n !\right)$ holds for small value of $|x|$ [42], we apply this approximation in (12) and can obtain the asymptotic outage probability of the DF relaying networks without cache as

$$
P_{\mathrm{out}}^{\mathrm{wo}} \simeq \frac{m_{1}^{m_{1}-1}}{\Gamma\left(m_{1}\right)}\left(\frac{\gamma_{1 t} \sigma^{2}}{P \alpha}\right)^{m_{1}}+\frac{m_{2}^{m_{2}-1}}{\Gamma\left(m_{2}\right)}\left(\frac{\gamma_{1 t} \sigma^{2}}{P \beta}\right)^{m_{2}} .
$$

By considering the relationship between $m_{1}$ and $m_{2}$, we can further specify the asymptotic outage probability as

$$
\begin{aligned}
& P_{\text {out }}^{\text {wo }} \\
& \simeq \begin{cases}\frac{m_{1}^{m_{1}-1}}{\Gamma\left(m_{1}\right)}\left(\frac{\gamma_{1 t} \sigma^{2}}{P \alpha}\right)^{m_{1}}, & \text { If } m_{1}<m_{2} \\
\frac{m_{1}^{m_{1}-1}}{\Gamma\left(m_{1}\right)}\left(\frac{\gamma_{1 t} \sigma^{2}}{P \alpha}\right)^{m_{1}}+\frac{m_{2}^{m_{2}-1}}{\Gamma\left(m_{2}\right)}\left(\frac{\gamma_{1 t} \sigma^{2}}{P \beta}\right)^{m_{2}}, & \text { If } m_{1}=m_{2} \\
\frac{m_{2}^{m_{2}-1}}{\Gamma\left(m_{2}\right)}\left(\frac{\gamma_{1 t} \sigma^{2}}{P \beta}\right)^{m_{2}}, & \text { If } m_{1}>m_{2} .\end{cases}
\end{aligned}
$$

From this asymptotic expression, we can find that the weaker hop between the dual hops becomes the bottleneck of the system whole transmission, and the system diversity order is equal to $\min \left(m_{1}, m_{2}\right)$. In particular, if the first hop is weaker with $m_{1}<m_{2}$, the first hop regulates the system transmission performance and the diversity order is $m_{1}$; if the second hop is weaker with $m_{2}<m_{1}$, the second hop becomes the bottleneck of the system whole transmission and the diversity order is $m_{2}$.

3.1.2. With Cache. For the cache aided DF relaying networks, the destination can directly access the data from the relays, 
and the outage event occurs when the $n^{*}$ th relay is selected to assist the data transmission,

$$
\log _{2}\left(1+\mathrm{SNR}_{D}\right)<R_{t}
$$

which is equivalent to

$$
\mathrm{SNR}_{D}<\gamma_{2 t}
$$

where $\gamma_{2 t}=2^{R_{t}}-1$ is the SNR threshold with cache. From this expression, we can write the system outage probability as

$$
\begin{aligned}
P_{\text {out }}^{\mathrm{w}} & =\operatorname{Pr}\left(\mathrm{SNR}_{D}<\gamma_{2 t}\right) \\
& =\operatorname{Pr}\left(v_{1}<\frac{\gamma_{2 t} \sigma^{2}}{P}, \ldots, v_{N}<\frac{\gamma_{2 t} \sigma^{2}}{P}\right) .
\end{aligned}
$$

By considering that $v_{n}$ values are independent of each other and are identically distributed, we can further write $P_{\text {out }}^{\mathrm{w}}$ as

$$
P_{\text {out }}^{\mathrm{W}}=[\underbrace{\operatorname{Pr}\left(v_{1}<\frac{\gamma_{2 t} \sigma^{2}}{P}\right)}_{I}]^{N} .
$$

To compute the probability $I$, we apply the PDF of $f_{v_{1}}(v)=$ $\left(m_{2}^{m_{2}} v^{m_{2}-1} / \beta^{m_{2}} \Gamma\left(m_{2}\right)\right) e^{-m_{2} v / \beta}$ as

$$
\begin{aligned}
I & =\int_{0}^{\gamma_{2 t} \sigma^{2} / P} \frac{m_{2}^{m_{2}} v^{m_{2}-1}}{\beta^{m_{2}} \Gamma\left(m_{2}\right)} e^{-m_{2} v / \beta} d v \\
& =1-e^{-m_{2} \gamma_{2 t} \sigma^{2} / P \beta} \sum_{k=0}^{m_{2}-1} \frac{1}{k !}\left(\frac{m_{2} \gamma_{2 t} \sigma^{2}}{P \beta}\right)^{k} .
\end{aligned}
$$

Accordingly, we obtain the analytical expression of outage probability for the cache aided DF relaying networks as

$$
P_{\text {out }}^{\mathrm{w}}=\left(1-e^{-m_{2} \gamma_{2 t} \sigma^{2} / P \beta} \sum_{k=0}^{m_{2}-1} \frac{1}{k !}\left(\frac{m_{2} \gamma_{2 t} \sigma^{2}}{P \beta}\right)^{k}\right)^{N} .
$$

Note that the above equation includes some elementary functions only and is also easy to be evaluated.
We now derive the asymptotic outage probability for the considered system, to obtain more insights on the system design. By applying the approximation of $e^{x} \simeq \sum_{n=0}^{N}\left(x^{n} / n !\right)$ into $I$, we can simplify the expression of $I$ as

$$
I \simeq \frac{m_{2}^{m_{2}-1}}{\Gamma\left(m_{2}\right)}\left(\frac{\gamma_{2 t} \sigma^{2}}{P \beta}\right)^{m_{2}} .
$$

From the asymptotic $I$, we can obtain the asymptotic expression of the outage probability for the cache aided DF relaying networks as

$$
P_{\text {out }}^{\mathrm{w}} \simeq\left(\frac{m_{2}^{m_{2}-1}}{\Gamma\left(m_{2}\right)}\right)^{N}\left(\frac{\gamma_{2 t} \sigma^{2}}{P \beta}\right)^{m_{2} N} .
$$

From this equation, we can find that the system diversity order is equal to $m_{2} N$, indicating that the system transmission performance can be rapidly improved by increasing the number of cache relays. Moreover, the system performance is not dependent on the first relaying links, as the destination can directly access the data from the relays.

3.2. SER. In this part, we derive the analytical expression of symbol error rate for the DF relaying networks, as well as the asymptotic expression in the high regime of transmit power. We use $P_{e}^{\text {wo }}$ and $P_{e}^{\mathrm{w}}$ to denote the SER of the system without or with cache, respectively. For the linear modulation scheme, the SER can be computed from the expression of outage probability [43]

$$
P_{e}=\frac{1}{\sqrt{2 \pi}} \int_{0}^{\infty} F_{\mathrm{SNR}_{D}}\left(\frac{x^{2}}{\lambda}\right) e^{-x^{2} / 2} d x
$$

where $F_{\mathrm{SNR}_{D}}(x)$ is the $\mathrm{CDF}$ of the received $\mathrm{SNR}$ at the destination $D$, which can be obtained from the analytical expression of outage probability in (12) and (20). And $\lambda$ is a constant depending on the specific modulation scheme. For example, $\lambda$ is equal to 2 when the BPSK modulation is used.

3.2.1. Without Cache. For the traditional dual-hop relaying networks without cache, we can write the system SRR as

$$
\begin{aligned}
P_{e}^{\mathrm{wo}} & =\frac{1}{\sqrt{2 \pi}} \int_{0}^{\infty}\left[1-e^{-\left(x^{2} \sigma^{2} / P \lambda\right)\left(m_{1} / \alpha+m_{2} / \beta\right)} \sum_{k_{1}=0}^{m_{1}-1} \sum_{k_{2}=0}^{m_{2}-1} \frac{1}{k_{1} ! k_{2} !}\left(\frac{m_{1} x^{2} \sigma^{2}}{P \alpha \lambda}\right)^{k_{1}}\left(\frac{m_{2} x^{2} \sigma^{2}}{P \beta \lambda}\right)^{k_{2}}\right] e^{-x^{2} / 2} d x \\
& =\frac{1}{2}-\sum_{k_{1}=0}^{m_{1}-1} \sum_{k_{2}=0}^{m_{2}-1} \frac{1}{k_{1} ! k_{2} !}\left(\frac{m_{1} \sigma^{2}}{P \alpha \lambda}\right)^{k_{1}}\left(\frac{m_{2} \sigma^{2}}{P \beta \lambda}\right)^{k_{2}} \times \frac{1}{\sqrt{2 \pi}} \int_{0}^{\infty} x^{2\left(k_{1}+k_{2}\right)} e^{-\left[1 / 2+\left(\sigma^{2} / P \lambda\right)\left(m_{1} / \alpha+m_{2} / \beta\right)\right] x^{2}} d x \\
& =\frac{1}{2}-\sum_{k_{1}=0}^{m_{1}-1} \sum_{k_{2}=0}^{m_{2}-1} \frac{1}{k_{1} ! k_{2} !}\left(\frac{m_{1} \sigma^{2}}{P \alpha \lambda}\right)^{k_{1}}\left(\frac{m_{2} \sigma^{2}}{P \beta \lambda}\right)^{k_{2}} \times \frac{b_{k_{1}+k_{2}}}{\left[1+\left(2 \sigma^{2} / P \lambda\right)\left(m_{1} / \alpha+m_{2} / \beta\right)\right]^{k_{1}+k_{2}+1 / 2}},
\end{aligned}
$$


where [42, eq. (3.321.3)] and [42, eq. (3.461.2)] are applied and $b_{n}$ is

$$
b_{n}= \begin{cases}1, & \text { If } n=0 \\ (2 n-1) ! !, & \text { If } n \geq 1\end{cases}
$$

Note that the analytical expression of SER in (25) contains some elementary functions only, and hence we can easily evaluate the system SER for the traditional DF relaying networks without cache aid. The details about the numerical computation can be found in [44-46].

We now propose to derive the asymptotic SER for the traditional DF relaying networks without cache, in the high regime of transmit power $P$. By combining (13) and (23), we can write the asymptotic SER with high transmit power $P$ as

$$
\begin{aligned}
& P_{e}^{\mathrm{wo}} \simeq \frac{1}{\sqrt{2 \pi}} \\
& \cdot \int_{0}^{\infty}\left[\frac{m_{1}^{m_{1}-1}}{\Gamma\left(m_{1}\right)}\left(\frac{x^{2} \sigma^{2}}{P \alpha \lambda}\right)^{m_{1}}+\frac{m_{2}^{m_{2}-1}}{\Gamma\left(m_{2}\right)}\left(\frac{x^{2} \sigma^{2}}{P \beta \lambda}\right)^{m_{2}}\right] \\
& \cdot e^{-x^{2} / 2} d x=\frac{m_{1}^{m_{1}-1}\left(2 m_{1}-1\right) ! !}{2 \Gamma\left(m_{1}\right)} \frac{1}{(P \alpha \lambda)^{m_{1}}} \\
&+\frac{m_{2}^{m_{2}-1}\left(2 m_{2}-1\right) ! !}{2 \Gamma\left(m_{2}\right)} \frac{1}{(P \beta \lambda)^{m_{2}}},
\end{aligned}
$$

where [42, eq. (3.461.2)] is used in the last equality. From this asymptotic SER, we can find that the system diversity order is equal to $\min \left(m_{1}, m_{2}\right)$, indicating that the weaker hop between dual hops becomes the bottleneck of the whole data transmission. In particular, the bottleneck is the first hop link if $m_{1}<m_{2}$ and the second hop otherwise.

3.2.2. With Cache. To compute the analytical expression of SER for the cache aided DF relaying networks, we first reexpress the analytical expression in (12) by using the binomial expansion [42],

$$
P_{\text {out }}^{\mathrm{w}}=\widetilde{\sum} \frac{c_{1 n}}{c_{2 n}} e^{-j m_{2} \gamma_{2 t} \sigma^{2} / P \beta}\left(\frac{m_{2} \gamma_{2 t} \sigma^{2}}{P \beta}\right)^{c_{3 n}},
$$

where

$$
\begin{aligned}
\widetilde{\sum}= & \sum_{j=0}^{N} \sum_{n_{1}=0}^{j} \sum_{n_{2}=0}^{n_{1}} \cdots \sum_{n_{m_{2}-1}=0}^{m_{2}-2}, \\
c_{1 n}= & \left(\begin{array}{c}
j \\
n_{1}
\end{array}\right)\left(\begin{array}{l}
n_{1} \\
n_{2}
\end{array}\right) \cdots\left(\begin{array}{c}
n_{m_{2}-2} \\
n_{m_{2}-1}
\end{array}\right), \\
c_{2 n}= & (-1)^{j}(2 !)^{n_{2}-n_{3}}(3 !)^{n_{3}-n_{4}} \cdots \\
& \times\left(\left(m_{2}-2\right) !\right)^{n_{m_{2}-2}-n_{m_{2}-1}}\left(\left(m_{2}-1\right) !\right)^{n_{m_{2}-1}} \\
c_{3 n}= & n_{1}+n_{2}+\cdots+n_{m_{2}-1} \cdot
\end{aligned}
$$

From this expression, we write the system SER for the cache aided relaying networks as

$$
\begin{aligned}
P_{e}^{\mathrm{W}} & =\widetilde{\sum} \frac{c_{1 n}}{\sqrt{2 \pi} c_{2 n}}\left(\frac{m_{2} \sigma^{2}}{P \beta \lambda}\right)^{c_{3 n}} \\
& \cdot \int_{0}^{\infty} e^{-\left(1 / 2+j m_{2} \sigma^{2} / P \beta \lambda\right) x^{2}} x^{2 c_{3 n}} d x \\
= & \widetilde{\sum} \frac{c_{1 n}}{2 c_{2 n}}\left(\frac{m_{2} \sigma^{2}}{P \beta \lambda}\right)^{c_{3 n}} \frac{b_{c_{3 n}}}{\left(1+2 j m_{2} \sigma^{2} / P \beta \lambda\right)^{c_{3 n}+1 / 2}},
\end{aligned}
$$

where [42, eq. (3.321.3)] and [42, eq. (3.461.2)] are used. From this analytical expression, we can easily evaluate the system SER for the DF relaying networks without cache.

We now propose to derive the asymptotic SER for the cache aided DF relaying networks, where we assume that the transmit power is large. By applying the asymptotic outage probability of (22) in (23), we can obtain the asymptotic SER as

$$
\begin{aligned}
P_{e}^{\mathrm{W}} & \simeq \frac{1}{\sqrt{2 \pi}} \int_{0}^{\infty}\left(\frac{m_{2}^{m_{2}-1}}{\Gamma\left(m_{2}\right)}\right)^{N}\left(\frac{x^{2} \sigma^{2}}{P \beta} \lambda\right)^{m_{2} N} e^{-x^{2} / 2} d x \\
& =\frac{\left(2 m_{2} N-1\right) ! !}{2}\left(\frac{m_{2}^{m_{2}-1}}{\Gamma\left(m_{2}\right)}\right)^{N} \frac{1}{(P \beta \lambda)^{m_{2} N}} .
\end{aligned}
$$

From this asymptotic SER, we can find that the system diversity order is equal to $m_{2} N$, indicating that the system transmission performance can be rapidly improved by increasing the fading parameter $m_{2}$ or the number of relays.

\section{Simulation and Numerical Results}

In this section, we provide some numerical and simulation results to validate the proposed studies. All links in the network experience Nakagami-m fading, and we set $\alpha=\beta=$ 1 without loss of generality. The power of the additive white noise at the receiver is set to unity with $\sigma=1$. The target data rate $R_{t}$ is set to $1 \mathrm{bps} / \mathrm{Hz}$, so that the two SNR thresholds $\gamma_{1 t}$ and $\gamma_{2 t}$ are associated with 3 and 1, respectively. For the SER computation, the BPSK modulation scheme is used, so that the modulation constant $\lambda$ is set to 2 .

Figure 2 depicts the outage probability of the traditional dual-hop relaying networks versus the transmit power $P$, where $m_{1}=2$ and $m_{2}$ varies from 1 to 3 . As observed from this figure, we can find that, for various values of $P$ and $m_{2}$, the analytical result matches well with the simulation value and the asymptotic result converges to the exact one in the high regime of transmit power $P$. This validates the effectiveness of the derived analytical and asymptotic expressions of outage probability for the traditional dual-hop relaying networks without cache. Moreover, the curve slope of the outage probability linearly increases with $m_{2}$, when $m_{2} \leq m_{1}$, since the second relaying link becomes the bottleneck of the system data transmission. In further, the curves with $m_{2}=2$ and $m_{2}=3$ have the same slope, indicating that the system diversity order remains unchanged. 


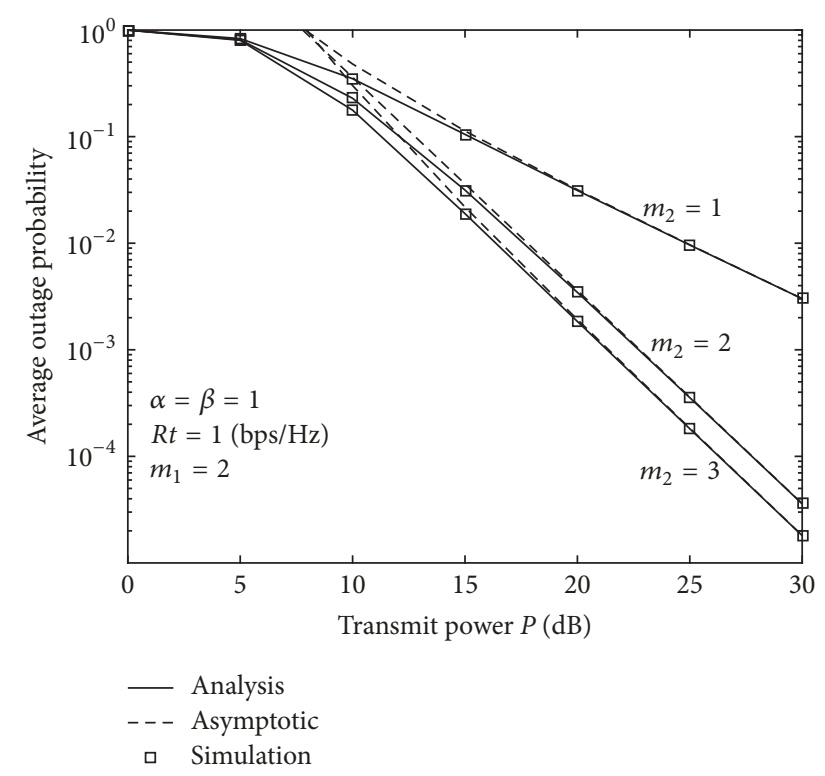

FIGURE 2: Outage probability versus the transmit power $P$ without cache.

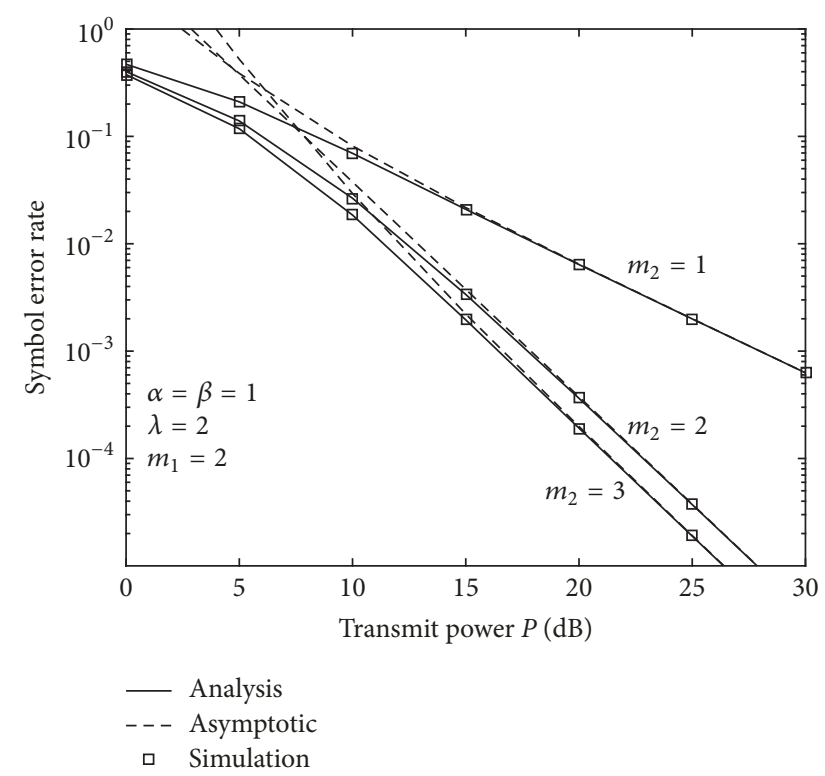

Figure 3: Symbol error rate versus the transmit power $P$ without cache.

Figure 3 demonstrates the symbol error rate of the traditional dual-hop relaying networks without cache, where $\lambda=2$, the transmit power $P$ varies from $0 \mathrm{~dB}$ to $30 \mathrm{~dB}$, and $m_{2}$ varies from 1 to 3 . We can see from this figure that the analytical SER fits well with the simulated SER and the asymptotic SER converges to the exact SER in the high region of transmit power $P$. This verifies the derived analytical and asymptotic expressions of SER for the traditional dualhop relaying networks without cache. Moreover, the curve slope increases with $m_{2}$ when $m_{2} \leq m_{1}$, since the second relaying link becomes the bottleneck of the system whole data

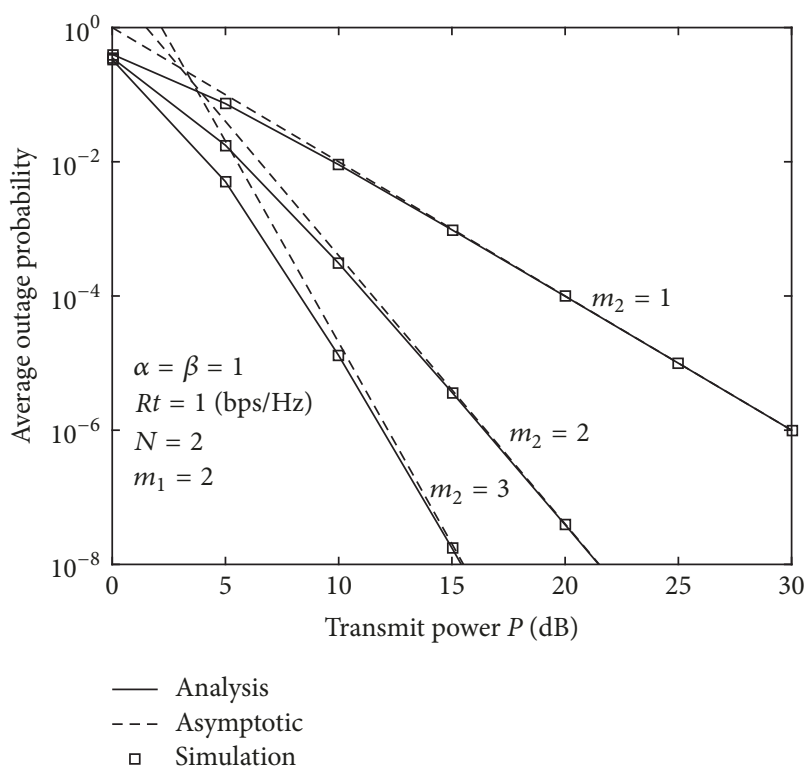

Figure 4: Outage probability versus the transmit power $P$ with cache.

transmission. Furthermore, the curve slope with $m_{2}=2$ is the same as that of $m_{2}=3$, indicating that the system diversity order remains unchanged.

Figure 4 illustrates the outage probability of the cache aided DF relaying networks, where $N=2, P$ varies from $0 \mathrm{~dB}$ to $30 \mathrm{~dB}$, and $m_{2}$ varies from 1 to 3 . As seen in Figure 4 , we can find that the analytical outage probability tallies with the simulation probability and the asymptotic outage probability converges to the exact $P_{\text {out }}$ with large value of $P$. This validates the correctness of the derived analytical and asymptotic expressions of outage probability. Moreover, the curve slopes are in parallel with the value of $m_{2}$, indicating that the system diversity order increases with the value of $m_{2}$. By comparing Figures 2 and 4, we can obtain that, for achieving a target level of outage probability $10^{-4}$, the transmit power can be saved by about $17.5 \mathrm{~dB}$ when $m_{2}=3$, indicating that the cache technique can save the power resource efficiently.

Figure 5 provides the symbol error rate of the cache aided DF relaying networks versus the transmit power $P$, where $\lambda=2$ and $m_{1}=2$ and $m_{2}$ varies from 1 to 3 . We can see from Figure 5 that the analytical SER is identical to the simulation one, and the asymptotic SER converges to the exact value in the high regime of transmit power $P$. This verifies the derived analytical and asymptotic expressions of SER for the cache aided DF relaying networks. Moreover, the curve slopes are in parallel with the value of $m_{2}$, indicating that the system diversity order increases linearly with $m_{2}$. By comparing Figures 3 and 5, we can find that, to achieve a target SER level of $10^{-4}$, using cache can save about $10 \mathrm{~dB}$, indicating that cache will be very useful in the scarce power resource scenarios.

Figures 6 and 7 show the impact of number of relays $N$ on the performance of cache aided relaying networks versus the transmit power $P$, where $m_{1}=m_{2}=2$ and $N$ varies from 1 to 3. Specifically, Figures 6 and 7 are associated with 


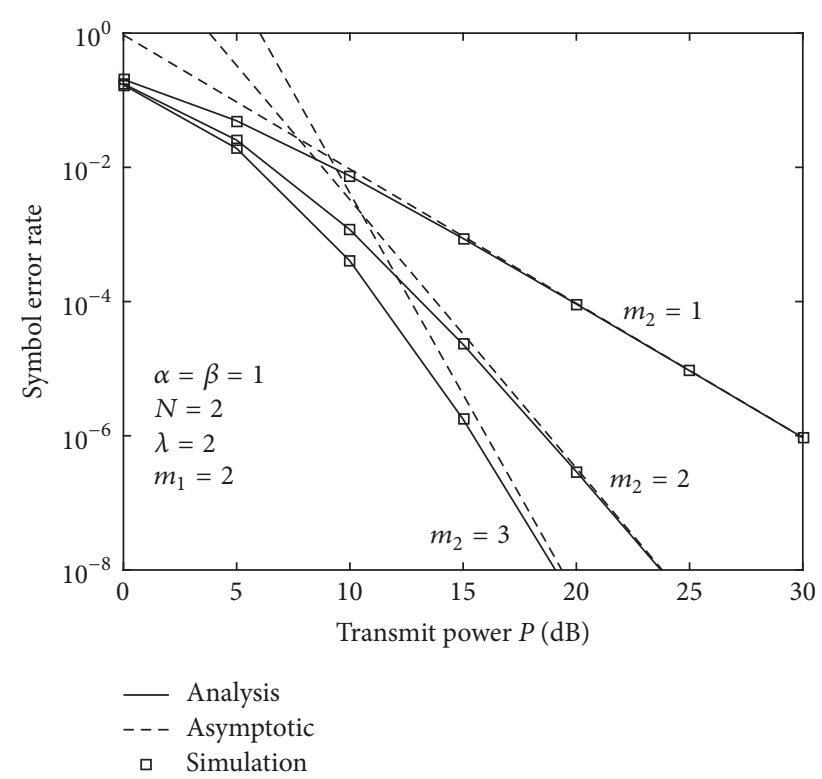

FIGURE 5: Symbol error rate versus the transmit power $P$ with cache.

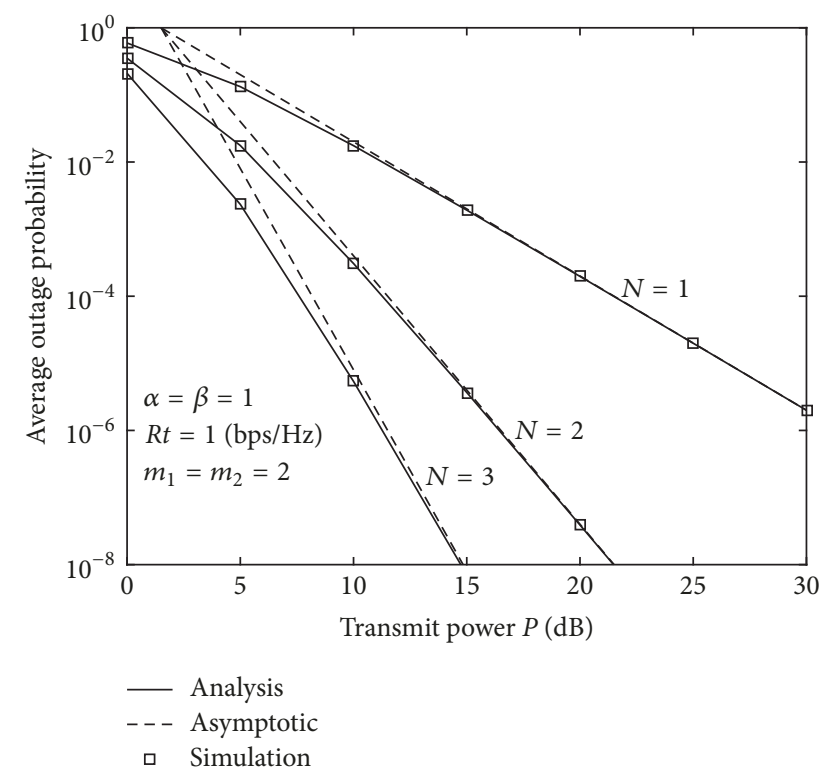

FIGURE 6: Impact of relay number $N$ on the cache aided relaying networks: outage probability.

outage probability and SER, respectively. We can find that, for different values of $N$, the analytical performance is in good agreement with the simulation value and the asymptotic value converges to the exact one when the transmit power is large, which validates the effectiveness of the derived analytical and asymptotic expressions of outage probability and SER. Moreover, the curve slopes are in parallel with $N$, indicating that the system diversity order increases linearly with the number of relays. For the target outage probability and SER around $10^{-4}$, we can find that the cache technique with $N=3$

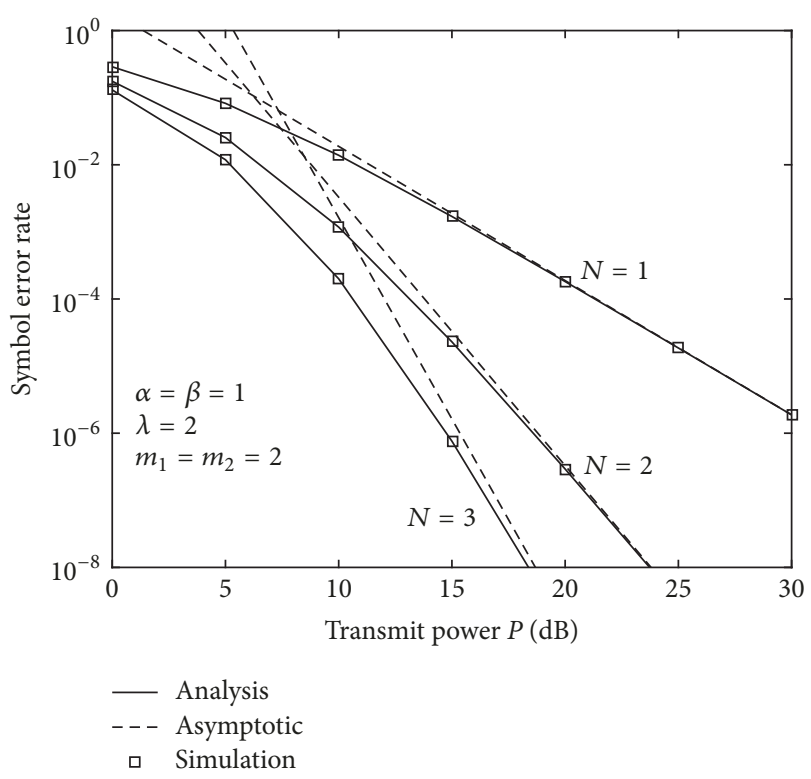

FigURE 7: Impact of relay number $N$ on the cache aided relaying networks: symbol error rate.

can save about $18.5 \mathrm{~dB}$ and $11 \mathrm{~dB}$, respectively, indicating that cache can be used to save power resource.

\section{Conclusions}

This paper studied a two-hop DF relaying network, where cache could be used to prefetch the data from the source to the nodes around the destination. In this way, the destination could directly access the data from the relay nodes, instead of communicating with the source. For the DF relaying networks without and with cache, the system performances were studied by deriving the analytical expressions of outage probability and symbol error rate (SER). The asymptotic outage probability and SER were also provided in the high regime of transmit power, from which we could find the system diversity order is rapidly increased by using cache and the system performance is significantly improved. Simulation and numerical results were demonstrated to verify the proposed studies, and the transmit power could be saved by about $18.5 \mathrm{~dB}$ and $11 \mathrm{~dB}$ for achieving outage probability and SER level of $10^{-4}$.

\section{Conflicts of Interest}

The authors declare that they have no conflicts of interest.

\section{Acknowledgments}

This paper was supported by the Innovation Team Project of Guangdong Province University under Grant 2016KCXTD017 and by the NSF of China under Grant 61501127, NSF of Guangdong under Grant 2016A030313705, and Special Funds for Applied S\&T of Guangdong under Grants 2015B010129001, 2014B090907010, and 2015B010106010. 


\section{References}

[1] J. Zhao, S. Ni, and Y. Gong, "Peak-to-Average Power Ratio Reduction of FBMC/OQAM Signal Using a Joint Optimization Scheme," IEEE Access, vol. 5, pp. 15810-15819, 2017.

[2] X. Xing, D. Xie, and G. Wang, "Energy-balanced data gathering and aggregating in wsns: a compressed sensing scheme," International Journal of Distributed Sensor Networks, vol. 2015, Article ID 585191, 10 pages, 2015.

[3] X. Liu, F. Li, and Z. Na, "Optimal Resource Allocation in Simultaneous Cooperative Spectrum Sensing and Energy Harvesting for Multichannel Cognitive Radio," IEEE Access, vol. 5, pp. 38013812, 2017.

[4] S. Ni, J. Zhao, and Y. Gong, "Optimal pilot design in massive MIMO systems based on channel estimation," IET Communications, vol. 11, no. 7, pp. 975-984, 2017.

[5] X. Liu, M. Jia, X. Gu, and X. Tan, "Optimal periodic cooperative spectrum sensing based on weight fusion in cognitive radio networks," Sensors, vol. 13, no. 4, pp. 5251-5272, 2013.

[6] D. Tang, D. Jiang, G. Huang, and M. Xia, "Energy states aided relay selection and optimal power allocation for cognitive relaying networks," IET Communications, vol. 11, no. 7, pp. 10451052, 2017.

[7] Z. Sun, L. Li, X. Li, X. Xing, and Y. Li, "Optimization coverage conserving protocol with authentication in wireless sensor networks," International Journal of Distributed Sensor Networks, vol. 13, no. 3, 2017.

[8] X. Liu, K. Chen, J. Yan, and Z. Na, “Optimal Energy Harvestingbased Weighed Cooperative Spectrum Sensing in Cognitive Radio Network," Mobile Networks and Applications, vol. 21, no. 6, pp. 908-919, 2016.

[9] Z. Sun, C. Li, X. Xing, H. Wang, B. Yan, and X. Li, "K-degree coverage algorithm based on optimization nodes deployment in wireless sensor networks," International Journal of Distributed Sensor Networks, vol. 13, no. 2, 2017.

[10] X. Liu, M. Jia, and X. Tan, "Threshold optimization of cooperative spectrum sensing in cognitive radio networks," Radio Science, vol. 48, no. 1, pp. 23-32, 2013.

[11] L. Dong, Z. Han, A. P. Petropulu, and H. . Poor, "Improving wireless physical layer security via cooperating relays," IEEE Transactions on Signal Processing, vol. 58, no. 3, part 2, pp. 18751888, 2010.

[12] L. Fan, R. Zhao, F.-K. Gong, N. Yang, and G. K. Karagiannidis, "Secure Multiple Amplify-and-Forward Relaying over Correlated Fading Channels," IEEE Transactions on Communications, vol. 65, no. 7, pp. 2811-2820, 2017.

[13] T. Beniero, S. Redana, J. Hämäläinen, and B. Raaf, "Effect of relaying on coverage in 3GPP LTE-advanced," in Proceedings of the VTC Spring 2009 - IEEE 69th Vehicular Technology Conference, pp. 1-5, April 2009.

[14] L. Xiao, T. E. Fuja, and D. J. Costello Jr., "Mobile relaying: Coverage extension and throughput enhancement," IEEE Transactions on Communications, vol. 58, no. 9, pp. 2709-2717, 2010.

[15] C. Zhong, S. Jin, and K.-K. Wong, "Dual-hop systems with noisy relay and interference-limited destination," IEEE Transactions on Communications, vol. 58, no. 3, pp. 764-768, 2010.

[16] A. Shah and A. M. Haimovich, "Performance analysis of optimum combining in wireless communications with rayleigh fading and cochannel interference," IEEE Transactions on Communications, vol. 46, no. 4, pp. 473-479, 1998.
[17] G. Zhu, C. Zhong, H. A. Suraweera, Z. Zhang, C. Yuen, and R. Yin, "Ergodic capacity comparison of different relay precoding schemes in dual-hop AF systems with co-channel interference," IEEE Transactions on Communications, vol. 62, no. 7, pp. 23142328, 2014.

[18] G. Huang and D. Tang, "Wireless Information and Power Transfer in Two-Way OFDM Amplify-and-Forward Relay Networks," IEEE Communications Letters, vol. 20, no. 8, pp. 1563-1566, 2016.

[19] P. He, Z. Deng, H. Wang, and Z. Liu, "Model approach to grammatical evolution: theory and case study," Soft Computing, vol. 20, no. 9, pp. 3537-3548, 2016.

[20] D. Lee, "SER of TAS-MRC with relay and user selection in MIMO-relay systems over non-identical nakagami fading channels," IEEE Communications Letters, vol. 21, no. 7, pp. 16451648, 2017.

[21] W. Tan, M. Matthaiou, S. Jin, and X. Li, "Spectral Efficiency of DFT-Based Processing Hybrid Architectures in Massive MIMO," IEEE Wireless Communications Letters, 2017.

[22] W. Tan, S. Jin, C. Wen, and T. Jiang, "Spectral efficiency of multi-user millimeter wave systems under single path with uniform rectangular arrays," EURASIP Journal on Wireless Communications and Networking, vol. 2017, no. 1, 2017.

[23] M. A. Maddah-Ali and U. Niesen, "Fundamental limits of caching," Institute of Electrical and Electronics Engineers Transactions on Information Theory, vol. 60, no. 5, pp. 2856-2867, 2014.

[24] M. Mohammadi Amiri and D. Gunduz, "Fundamental Limits of Coded Caching: Improved Delivery Rate-Cache Capacity Tradeoff," IEEE Transactions on Communications, vol. 65, no. 2, pp. 806-815, 2017.

[25] M. Tao, E. Chen, H. Zhou, and W. Yu, "Content-Centric Sparse Multicast Beamforming for Cache-Enabled Cloud RAN," IEEE Transactions on Wireless Communications, vol. 15, no. 9, pp. 6118-6131, 2016.

[26] G. Zheng, H. A. Suraweera, and I. Krikidis, "Optimization of hybrid cache placement for collaborative relaying," IEEE Communications Letters, vol. 21, no. 2, pp. 442-445, 2017.

[27] G. Liu, H. Liu, H. Chen, C. Zhou, and L. Shu, "Position-based adaptive quantization for target location estimation in wireless sensor networks using one-bit data," Wireless Communications and Mobile Computing, 2015.

[28] W. Chen, H. Lei, and K. Qi, "Lattice-based linearly homomorphic signatures in the standard model," Theoretical Computer Science, vol. 634, pp. 47-54, 2016.

[29] W. Yang, G. Wang, M. Z. A. Bhuiyan, and K.-K. R. Choo, "Hypergraph partitioning for social networks based on information entropy modularity," Journal of Network and Computer Applications, vol. 86, pp. 59-71, 2017.

[30] Y. Li, Z. Peng, D. Liang, H. Chang, and Z. Cai, "Facial age estimation by using stacked feature composition and selection," The Visual Computer, vol. 32, no. 12, pp. 1525-1536, 2016.

[31] N. Zhao, X. Liu, F. R. Yu, M. Li, and V. C. M. Leung, "Communications, caching, and computing oriented small cell networks with interference alignment," IEEE Communications Magazine, vol. 54, no. 9, pp. 29-35, 2016.

[32] W. Chen, L. Peng, J. Wang et al., "Inapproximability results for the minimum integral solution problem with preprocessing over infinity norm," Theoretical Computer Science, vol. 478, pp. 127-131, 2013. 
[33] Y. Gao, X. Shan, Z. Hu, D. Wang, Y. Li, and X. Tian, "Extended compressed tracking via random projection based on MSERs and online LS-SVM learning," Pattern Recognition, vol. 59, pp. 245-254, 2016.

[34] G. Liu, B. Xu, H. B. Chen, C. Zhang, and X. Hu, "Energyefficient scheduling for distributed estimation inwireless sensor and actuator networks with kriging," Ad Hoc \& Sensor Wireless Networks, vol. 27, no. 3-4, pp. 197-222, 2015.

[35] G. Pan, H. Lei, Y. Deng et al., "On Secrecy Performance of MISO SWIPT Systems with TAS and Imperfect CSI," IEEE Transactions on Communications, vol. 64, no. 9, pp. 3831-3843, 2016.

[36] L. Fan, X. Lei, N. Yang, T. Q. Duong, and G. K. Karagiannidis, "Secure Multiple Amplify-and-Forward Relaying with Cochannel Interference," IEEE Journal of Selected Topics in Signal Processing, vol. 10, no. 8, pp. 1494-1505, 2016.

[37] H. Shen, C. Gao, D. He, and L. Wu, "New biometrics-based authentication scheme for multi-server environment in critical systems," Journal of Ambient Intelligence and Humanized Computing, vol. 6, no. 6, pp. 825-834, 2015.

[38] W. Chen, N. F. Samatova, M. . Stallmann, W. Hendrix, and W. Ying, "On size-constrained minimum $s$ - $t$ cut problems and sizeconstrained dense subgraph problems," Theoretical Computer Science, vol. 609, no. part 2, pp. 434-442, 2016.

[39] G. Liu, J. Yao, Y. Liu, H. Chen, and D. Tang, "Channel-Aware Adaptive Quantization Method for Source Localization in Wireless Sensor Networks," International Journal of Distributed Sensor Networks, vol. 2015, Article ID 214081, 2015.

[40] M. Z. A. Bhuiyan, J. Wu, G. Wang, T. Wang, and M. M. Hassan, "e-Sampling: Event-sensitive autonomous adaptive sensing and low-cost monitoring in networked sensing systems," ACM Transactions on Autonomous and Adaptive Systems (TAAS), vol. 12, no. 1, article no. 1, 2017.

[41] L. Fan, X. Lei, N. Yang, T. Q. Duong, and G. K. Karagiannidis, "Secrecy Cooperative Networks with Outdated Relay Selection over Correlated Fading Channels," IEEE Transactions on Vehicular Technology, vol. 66, no. 8, pp. 7599-7603, 2017.

[42] I. S. Gradshteyn and I. M. Ryzhik, Table of integrals, series, and products, Academic Press, Inc., San Diego, CA, USA, 7th edition, 1996.

[43] G. Huang and W. Tu, "Optimal resource allocation in wirelesspowered OFDM relay networks," Computer Networks, vol. 104, pp. 94-107, 2016.

[44] X. Wang, X. Ma, and B. Bai, "Design of efficiently encodable nonbinary LDPC codes for adaptive coded modulation," Science China Information Sciences, vol. 57, no. 2, pp. 1-11, 2014.

[45] J. Yang, H. Wu, G. Huang, Y. Liang, and Y. Liao, "Modeling and coupling effect evaluation of thermal conductivity of ternary opacifier/fiber/aerogel composites for super-thermal insulation," Materials and Corrosion, vol. 133, pp. 224-236, 2017.

[46] X. Wang and S. Tang, "Bit-level soft-decision decoding of double and triple-parity reed-solomon codes through binary hamming code constraints," IEEE Communications Letters, vol. 19, no. 2, pp. 135-138, 2015. 


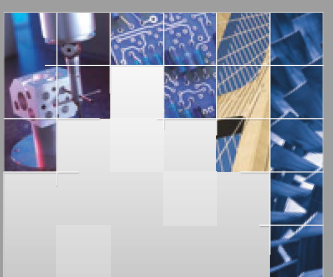

\section{Enfincering}
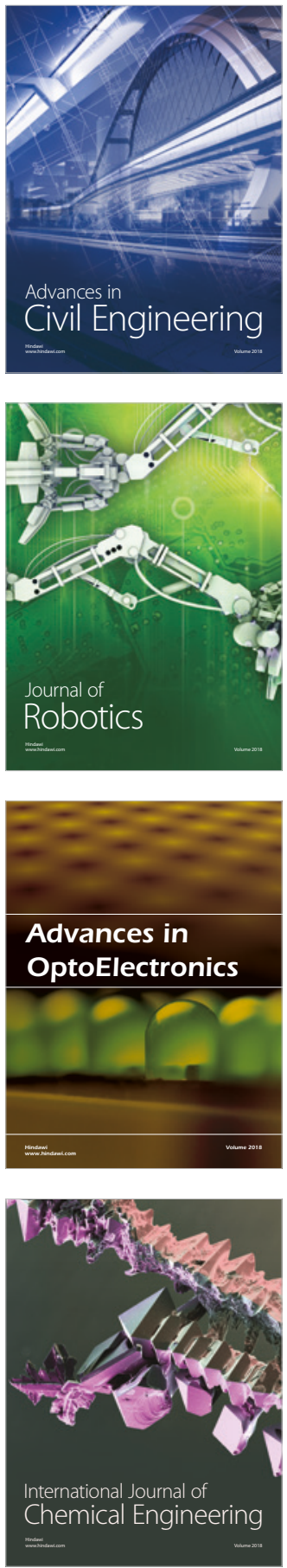

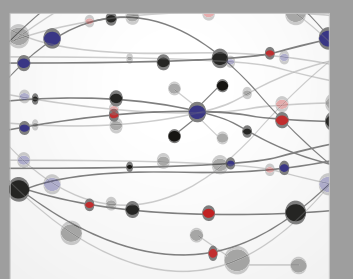

\section{Rotating \\ Machinery}

The Scientific World Journal

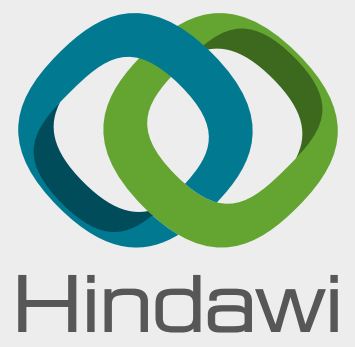

Submit your manuscripts at

www.hindawi.com
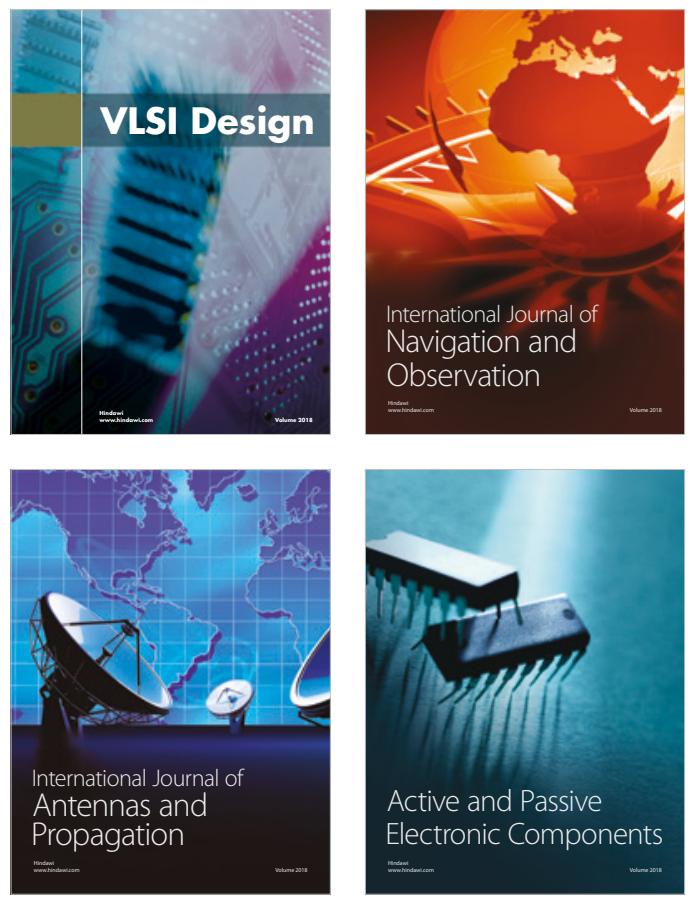
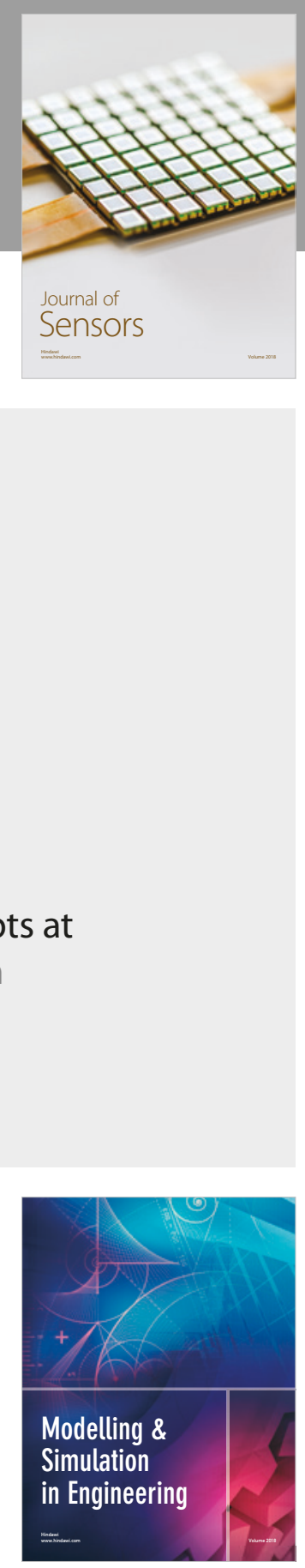

\section{Advances \\ Multimedia}
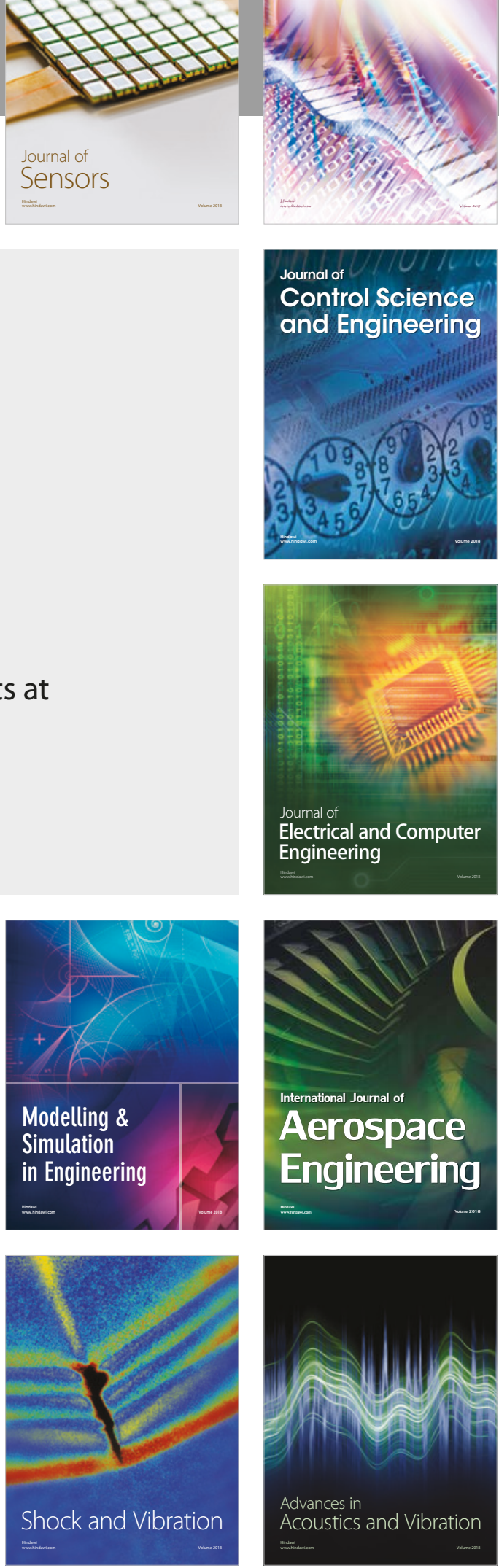